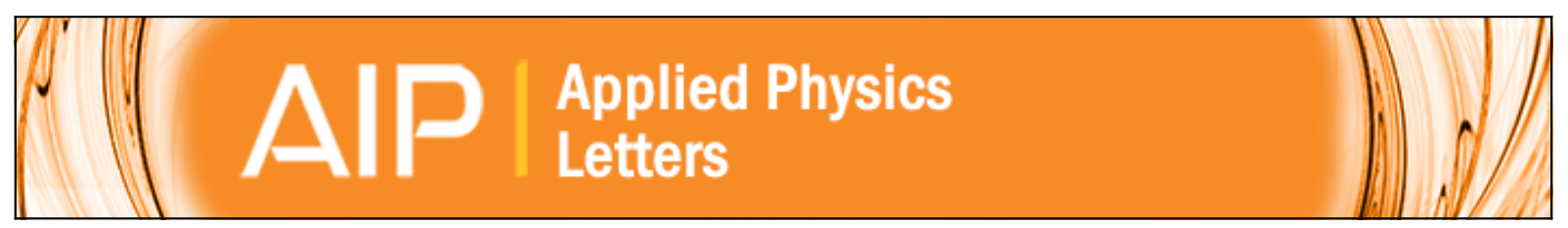

\title{
Tuning of noble metal work function with organophosphonate nanolayers
}

Ganpati Ramanath, Matthew Kwan, P. K. Chow, Y. Cardona Quintero, P. H. Mutin, and R. Ramprasad

Citation: Applied Physics Letters 105, 081601 (2014); doi: 10.1063/1.4890486

View online: http://dx.doi.org/10.1063/1.4890486

View Table of Contents: http://scitation.aip.org/content/aip/journal/apl/105/8?ver=pdfcov

Published by the AIP Publishing

\section{Articles you may be interested in}

Metal gate work function tuning by $\mathrm{Al}$ incorporation in TiN

J. Appl. Phys. 115, 074504 (2014); 10.1063/1.4866323

Enantioselectivity of (321) chiral noble metal surfaces: A density functional theory study of lactate adsorption J. Chem. Phys. 139, 224709 (2013); 10.1063/1.4839755

The canonical work function-strain relationship of the platinum metal: A first-principles approach to metal-gate transistor optimization

Appl. Phys. Lett. 102, 223504 (2013); 10.1063/1.4807939

Density functional theoretical study of pentacene/noble metal interfaces with van der Waals corrections: Vacuum level shifts and electronic structures

J. Chem. Phys. 132, 134703 (2010); 10.1063/1.3373389

Contact resistance between metal and carbon nanotube interconnects: Effect of work function and wettability Appl. Phys. Lett. 95, 264103 (2009); 10.1063/1.3255016

\section{A|P| $\begin{aligned} & \text { Journal of } \\ & \text { Applied Physics }\end{aligned}$}

Journal of Applied Physics is pleased to announce André Anders as its new Editor-in-Chief 


\title{
Tuning of noble metal work function with organophosphonate nanolayers
}

\author{
Ganpati Ramanath, ${ }^{1, \text { a) }}$ Matthew Kwan, ${ }^{1}$ P. K. Chow, ${ }^{1}$ Y. Cardona Quintero, ${ }^{2}$ P. H. Mutin, ${ }^{3}$ \\ and R. Ramprasad ${ }^{2}$ \\ ${ }^{1}$ Department of Materials Science and Engineering, Rensselaer Polytechnic Institute, Troy, \\ New York 12180, USA \\ ${ }^{2}$ Department of Materials Science and Engineering, University of Connecticut, Storrs, \\ Connecticut 06269, USA \\ ${ }^{3}$ Institut Charles Gerhardt Montpellier, UMR 5253 CNRS-IM2-ENSCM-UM1, Université Montpellier 2, \\ CC 1701, Place Eugène Bataillon, 34095 Montpellier Cedex 5, France
}

(Received 21 December 2013; accepted 6 July 2014; published online 25 August 2014)

\begin{abstract}
We demonstrate that weak chemical interactions between untethered moieties in molecular nanolayers on metal surfaces can strongly influence the effective work function $\Phi_{\text {eff. }}$ Electron spectroscopy shows that nanolayers of mercaptan-anchored organophosphonates on $\mathrm{Au}$ and $\mathrm{Pt}$ decrease $\Phi_{\text {eff }}$. The measured $\Phi_{\text {eff }}$ shifts correlate with the chemical state of phosphonic acid moieties, and scale with molecular length. These results are contrary to predictions of ab initio calculations of monolayer-capped surfaces, but are consistent with calculations of bilayer-capped surfaces with face-to-face hydrogen-bonded phosphonic acid moieties. Our findings indicate that intra-layer bonding and layering in molecular nanolayers can be key to tailoring heterointerfacial electronic properties for applications. (C) 2014 AIP Publishing LLC. [http://dx.doi.org/10.1063/1.4890486]
\end{abstract}

High-quality metal-oxide ${ }^{1,2}$ and metal-organic semiconductor $^{3,4}$ structures require tailored interfacial properties to ensure optimal functionality and reliability of advanced nanoelectronics devices. For example, it is crucial to tailor the band alignment of the gate metal and the gate insulators ${ }^{5}$ in metal-oxide-semiconductor devices. Appropriate doping of polysilicon metallization in devices with $\mathrm{SiO}_{2}$ gates allows the alignment of the metallization Fermi level with the desired band edge of the Si channel. The incompatibility of polysilicon with high dielectric permittivity oxides (e.g., $\mathrm{HfO}_{2}$ ) precludes this approach and requires the identification of metals that form stable interfaces with these oxides and devising strategies to tune the metal work function at the interface, or the effective work function $\left(\Phi_{\text {eff }}\right)$, to attain the desired band alignments.

Grafting molecular nanolayers (MNLs) with the appropriate termini, length, and structure between the metalinsulator interfaces is a potential means to tune $\Phi_{\text {eff }}$. Molecular adsorption is known to alter $\Phi_{\text {eff }}$ of metal ${ }^{6-10}$ and oxide $^{11-16}$ surfaces and interfaces, giving rise to shifts of $-1.9 \leq \Delta \Phi_{\text {eff }} \leq 1.5 \mathrm{eV}$ attributed to polar bonding, molecular length, and terminal moieties. For example, fostering electron transfer to a mercaptan anchor in alkanethiol-capped $\mathrm{Au}$ and/or larger dipole moment from a longer chain length can increase $\left|\Delta \Phi_{\text {eff }}\right|^{7,8,12}$ Dipole moment magnitude diminution via effects such as Pauli repulsion of surface electron charge densities $^{13,14}$ or by adjusting molecular coverage can decrease $\left|\Delta \Phi_{\text {eff }}\right|$. Organosilane and organophosphonate MNLs also offer the possibility of altering multiple interfacial properties, e.g., inhibit metal diffusion, ${ }^{17-19}$ improve adhesion $^{18-22}$ and thermal conductance, ${ }^{21,22}$ through interfacial bonding, and/or decomposition ${ }^{23}$ at temperatures above $300^{\circ} \mathrm{C}$. Additionally, organophosphonates are water-resilient

a)Email: Ramanath@rpi.edu and can improve electrical contact resistance and thin film transistor channel mobilities. ${ }^{24,25}$

Here, we show that the effective work function shift magnitudes $\left|\Delta \Phi_{\text {eff }}\right|$ on Au and Pt surfaces capped with MNLs of mercaptan-terminated organophosphonates correlates with the molecular length and chemical states of the phosphonic acid moieties. Our results suggest that intra-MNL layering via hydrogen bonding plays important roles that could be of potential use to tailoring $\Phi_{\text {eff }}$ for applications.

We deposited 50-nm-thick Au or Pt onto n-type $\mathrm{Si}(001)$ wafers with an 85 -nm-thick thermal oxide by e-beam evaporation in a $1.7 \times 10^{-7}$ Torr base pressure chamber at a rate of $2.0 \mathrm{~nm} / \mathrm{s}$. A 20-nm-thick Ti film was deposited on the $\mathrm{SiO}_{2}$ to promote adhesion with the noble metals. MNLs of 12-mercaptododecyl phosphonic acid (MDPA) and 3-mercaptopropyl phosphonic acid (MPPA) were adsorbed onto the metal by immersing the metalcoated wafers for $20 \mathrm{~h}$ in an ultrahigh purity $\mathrm{N}_{2}$-purged $1 \mathrm{mM}$ ethanolic solution at room temperature. The MNLfunctionalized metal films were rinsed in fresh ethanol, sonicated for $30 \mathrm{~s}$ and blown dry with $\mathrm{N}_{2}$, and placed on a $\sim 60^{\circ} \mathrm{C}$ hotplate for $30 \mathrm{~s}$.

We used ultraviolet photoelectron spectroscopy (UPS) to determine $\Phi_{\text {eff }}$ by subtracting the valence band width spanning the secondary electron energy onset edge $\mathrm{E}_{\mathrm{SEO}}$ and the Fermi level ${ }^{26} \mathrm{E}_{\mathrm{F}}$ from the incident photon energy $\mathrm{h} \nu$, i.e., $\Phi_{\text {eff }}=h \nu-\left(E_{\mathrm{F}}-E_{\mathrm{SEO}}\right)$. UPS spectra were acquired in a PHI 5400 system equipped with a $21.22 \mathrm{eV} \mathrm{He}^{\mathrm{I}}$ photon source at an analyzer pass energy of $5.85 \mathrm{eV}$. A $-9.00 \mathrm{~V}$ bias was applied to the metal to improve the $\mathrm{E}_{\mathrm{SEO}}$ edge resolution at low photoelectron kinetic energies. Core-level spectra were then recorded at a $23.5 \mathrm{eV}$ analyzer pass energy by carrying out X-ray photoelectron spectroscopy (XPS) using a $\mathrm{Al} \mathrm{K} \alpha$ probe beam at preselected surface-to-detector takeoff angles $\theta_{\text {sd }}$. We fit the background using the Shirley method. ${ }^{27}$ The C 1s $(284.8 \mathrm{eV})$ peak and $\mathrm{Au} 4 \mathrm{f}_{7 / 2}(84.0 \mathrm{eV})$ or $\mathrm{Pt} 4 \mathrm{f}_{7 / 2}$ 
$(71.1 \mathrm{eV})$ peaks were used as internal references for selfconsistently correcting for residual surface charging. MNL thicknesses were determined from the attenuation of the $\mathrm{Au}$ and Pt $4 \mathrm{f}_{7 / 2}$ and $4 \mathrm{~d}_{5 / 2}$ core-level peaks determined by XPS with decreasing $\theta_{\mathrm{sd}}$ by assuming an experimentally determined effective escape depth. ${ }^{28}$ The thicknesses extracted by this method agree well with values determined by optical ellipsometry by fixing the metal film optical constants and assuming an MNL refractive index of 1.5.

Density Functional Theory (DFT) calculations were carried out using the Vienna $a b$ initio simulation package (VASP) code, ${ }^{29,30}$ with the Perdew-Burke-Ernzerhof (PBE) generalized gradient approximation $(\mathrm{GGA})^{31}$ and the projector-augmented wave (PAW) ${ }^{32}$ approach. The metalMNL systems were modeled with seven layers of $(2 \times \sqrt{3})$ metal (111) surface, a $\mathrm{HS}\left(\mathrm{CH}_{2}\right)_{\mathrm{n}}-\mathrm{PO}(\mathrm{OH})_{2}$ molecule on top with $\mathrm{n}=2,3,4,5,6$, and 12 , and a vacuum region of $1.2 \mathrm{~nm}$. Three middle layers of the metal were fixed to their bulk positions. Monkhorst-Pack k-mesh of $5 \times 5 \times 1 \mathrm{k}$-points was used, and a dipole correction was applied to compensate for the asymmetric interface. The molecular tilt angle $\alpha$ formed at the anchoring sulfur atom was referenced to the surface normal. We computed the effect of molecular length on the work function by holding the molecular surface density constant. The $\Phi_{\text {eff }}$ of the MNL-capped surfaces were computed by matching the planar average local electrostatic potentials of the MNL-modified metal surface layer and the bulk metal layers, and taking the difference between the vacuum level and the Fermi energy. For clean $\mathrm{Au}(111)$ and $\mathrm{Pt}(111)$ surfaces, our computations yield work functions $\Phi_{\mathrm{Au}}=5.18 \mathrm{eV}$ and $\Phi_{\mathrm{Pt}}=5.70 \mathrm{eV}$, in agreement with previous theoretical calculations $\left(\Phi_{\mathrm{Au}}=5.15 \mathrm{eV}, \Phi_{\mathrm{Pt}}=5.69 \mathrm{eV}\right),{ }^{33}$ and experiments $\left(\Phi_{\mathrm{Au}}=5.35 \mathrm{eV}\right.$ and $\left.\Phi_{\mathrm{Pt}}=6.08 \pm 0.15 \mathrm{eV}\right) .{ }^{14,34}$

In our experiments, the MPPA MNL thickness increased with deposition time on Au substrates, but remained relatively unchanged on Pt, though, in both cases, the MNL thickness exceeded that expected from a monolayer. In addition, both MDPA and MPPA MNLs were thicker on Au than on $\mathrm{Pt}$ for the same deposition conditions, indicating the MNL formation kinetics is not the same on the two metals. Valence band spectra acquired from $\mathrm{Au}$ and Pt substrates with and without MNL (Fig. 1) show that both MPPA and MDPA MNLs alter $\Phi_{\text {eff }}$.

MNLs of MDPA, the longer molecule, decrease $\Phi_{\text {eff }}$ more than MNLs of MPPA. For both metals, $\left|\Delta \Phi_{\text {eff }}\right|$ is higher for greater MNL thickness (see Fig. 2). The experimentally measured $\Delta \Phi_{\text {eff }}$ for MPPA-capped Pt is within $0.2 \mathrm{eV}$ (the instrumental resolution) of that determined ab initio calculations for Pt capped with a MPPA monolayer. For MPPA-capped Au, our measurements show a $\Delta \Phi_{\text {eff }}$ that is $\sim 0.7 \mathrm{eV}$ lower than the calculated value for Au-capped with a MPPA monolayer. For MDPA-capped Au, the measured $\left|\Delta \Phi_{\text {eff }}\right|$ is more than $2 \mathrm{eV}$ lower than the calculated value. We could not theoretically determine $\Delta \Phi_{\text {eff }}$ for MDPA-capped $\mathrm{Pt}$ due to MDPA dissociation upon equilibration.

$A b$ initio calculations show an overall increase in $\Delta \Phi_{\text {eff }}$ for longer organophosphonate monolayers on $\mathrm{Au}$. In addition, molecules with an odd number of $\mathrm{CH}_{2}$ groups $(n)$ on Au yield a higher $\Phi_{\text {eff }}$ with lower tilt angle $\left(\alpha=26^{\circ}\right)$ than

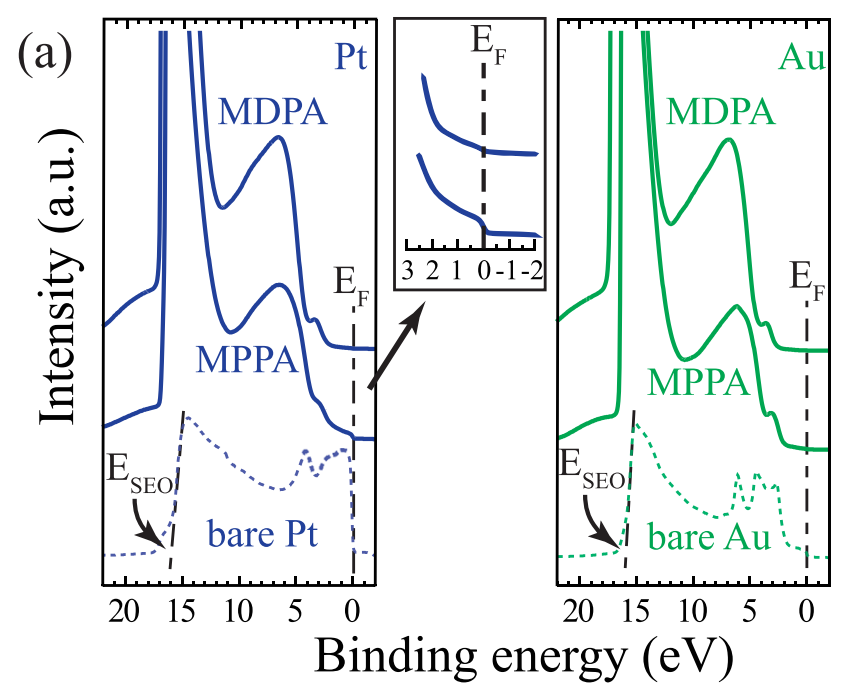

(b)

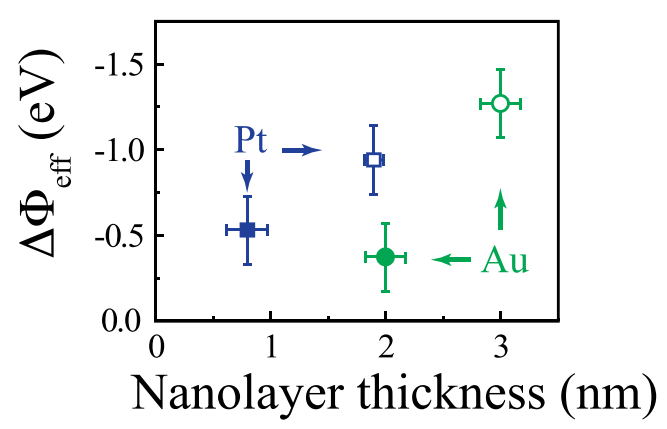

FIG. 1. (a) Representative valence band spectra from Pt (left) and Au (right) surfaces functionalized with MPPA and MDPA. Baseline spectra from sputter-cleaned pristine metal surfaces in vacuum (dashed curves), the locations of $E_{\mathrm{SEO}}$ the secondary electron onset energy, and $\mathrm{E}_{\mathrm{F}}$ the Fermi level are also shown. (b) Experimentally determined $\Delta \Phi_{\text {eff }}$ plotted as a function of measured nanolayer thickness for MPPA (filled symbols) and MDPA (unfilled symbols) on $\mathrm{Au}$ (circles) and Pt (squares).

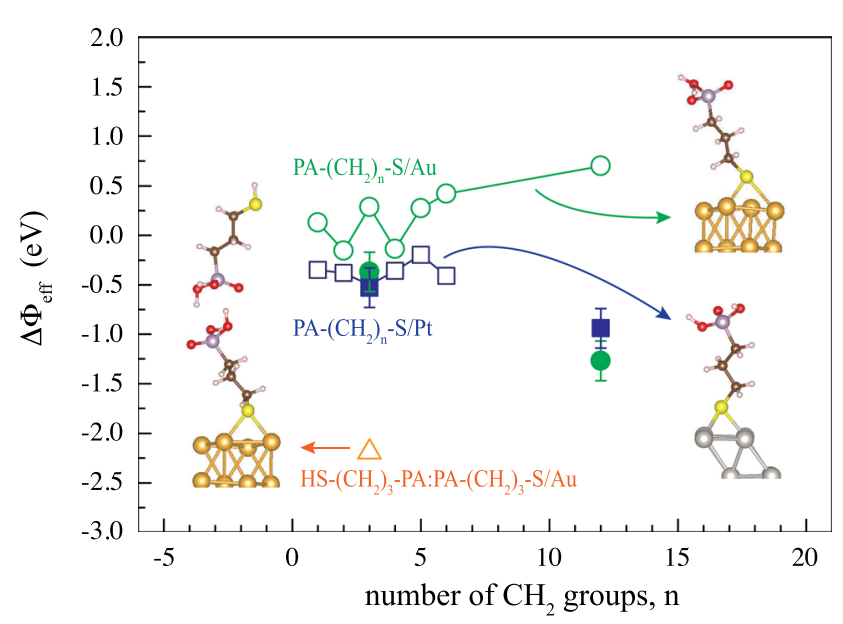

FIG. 2. Comparison of theoretically calculated (unfilled symbols) and experimentally determined (filled symbols) work function shifts for mercaptanterminated organophosphonates on $\mathrm{Au}$ (circles) and $\mathrm{Pt}$ (squares) plotted as a function of molecular length, lines are to guide the eye. The calculated $\Delta \Phi_{\text {eff }}$ for a MPPA bilayer, consisting of a chemisorbed sublayer on $\mathrm{Au}$, and a physisorbed overlayer held via hydrogen bonding between phosphonic acid moieties, is also shown (triangle). Schematic sketches of the theoretical model of chemisorbed MPPA on Pt (lower right), Au (upper right), and a MPPA overlayer physisorbed on to the chemisorbed layer via hydrogen bonding between phosphonic acid moieties (left). 
even $n$ with higher tilt angle $\left(\alpha=53^{\circ}\right)$. These results are consistent with the expectation that high molecular length and lower $\alpha$ increase the dipole moment along the surface normal pointing towards the Au surface. However, our experiments show an opposite trend with molecular length, indicating that the net dipole moment points away from the surface. We were, thus, persuaded to examine the intra-MNL molecular structure and bonding that may produce effects such as observed during inert gas adsorption. ${ }^{35} \mathrm{Ab}$ initio calculations of an Au surface capped with an MPPA bilayer held together via hydrogen bonding between the phosphonic acid moieties yield $\Delta \Phi_{\text {eff }}=-2.19 \mathrm{eV}$. The definitive agreement in the sign of $\Delta \Phi_{\text {eff }}$ with experiment underscores the role of intra-MNL hydrogen bonding and layering. The calculated $\left|\Delta \Phi_{\text {eff }}\right|$ exceeding the experimental values is not surprising considering that the simulation does not account for factors such as distributions of molecular orientations, bond strengths and disorder in the MNL.

In order to better understand the bonding and morphology within our MNLs, we examined core-level XPS spectra obtained from MPPA- and MDPA-treated metals at different takeoff angles $\theta_{\text {sd. }}$. For MPPA-treated Au (Fig. 3), we see two $\mathrm{O} 1 \mathrm{~s}$ sub-bands corresponding to $\mathrm{P}=\mathrm{O}(\sim 531.5 \mathrm{eV})$ and P-OH moieties $(\sim 532.5 \mathrm{eV})$ of the phosphonate termini ${ }^{36}$ (Fig. 3). We also see two S 2p sub-bands: from Au-S bonds at $\sim 162.0 \mathrm{eV}$ and unbound mercaptan at $\sim 163.5 \mathrm{eV} .^{36}$ The overall S 2 p peak shape is dependent on $\theta_{\text {sd }}$, pointing to different fractions of chemical states at different depths from the surface. For example, the Au-S sub-band is largest at high $\theta_{\text {sd }}$ indicative of chemisorbed $\mathrm{S}$ on $\mathrm{Au}$, and decreases significantly close to the surface (low $\theta_{\mathrm{sd}}$ ), where unbound mercaptan dominates. These results are consistent with results in Fig. 1(b), and indicate that in our experiments, the MPPA
MNL on Au is not a single ordered layer wherein all the molecules are directly tethered to the metal. Instead, the MPPA MNL consists of a physisorbed MPPA sub-layer on top of a chemisorbed MPPA sub-layer tethered to Au by Au-S bonds. We find a similar trend for MPPA on Pt and MDPA on Au and Pt (Fig. 4(a)), though MDPA on Pt has a steeper slope indicative of a thicker and/or more ordered physisorbed layer. The chemisorbed/physisorbed molecules ratio, estimated by comparing the bound and unbound $\mathrm{S}$ signals at high angles, is greater than $40 \%$, which is close to that expected from a bilayer with an above-mentioned configuration.

Removing the physisorbed MPPA from Au surfaces by rinsing the MPPA-coated surfaces with a $0.1 \mathrm{M} \mathrm{NaOH}$ solution followed by successive washing in $0.1 \mathrm{M} \mathrm{HCl}$ and water ${ }^{37}$ decreased the MNL thickness, decreased the S:Au intensity ratio 3 fold, but increased the Au-S sub-band intensity, and increased $\Delta \Phi_{\text {eff }}$ (Fig. 4(b)), bringing it closer to the calculated value for a MPPA bilayer on Au. However, the unbound mercaptan sub-band signature persists, and the MNL thickness remains greater than that of a monolayer. These suggest that washing helps MPPA multilayers approach a bilayer morphology.

While MNL coverage can alter $\Phi_{\text {eff }}$ by as much as $0.3 \mathrm{eV}$ on $\mathrm{Au}$ and $\mathrm{Si}$ surfaces, ${ }^{38-40}$ the $0.4 \mathrm{eV} \leq\left|\Delta \Phi_{\text {eff }}\right|$ $\leq 0.9 \mathrm{eV}$ measured in our experiments indicate that MNL coverage differences alone cannot explain the observed work function change. Photoelectron spectra showing a higher normalized S $2 \mathrm{p}$ core-level sub-band intensity corresponding to the metal-sulfur bond ${ }^{40}$ for MDPA-treated metal surfaces than that from corresponding surfaces treated with MPPA suggest a higher relative coverage. The relative S $2 p$ intensity is $2.8 \pm 1.6$ times larger for MDPA than MPPA for Pt surfaces, and $1.6 \pm 0.3$ times larger for $\mathrm{Au}$ surfaces.

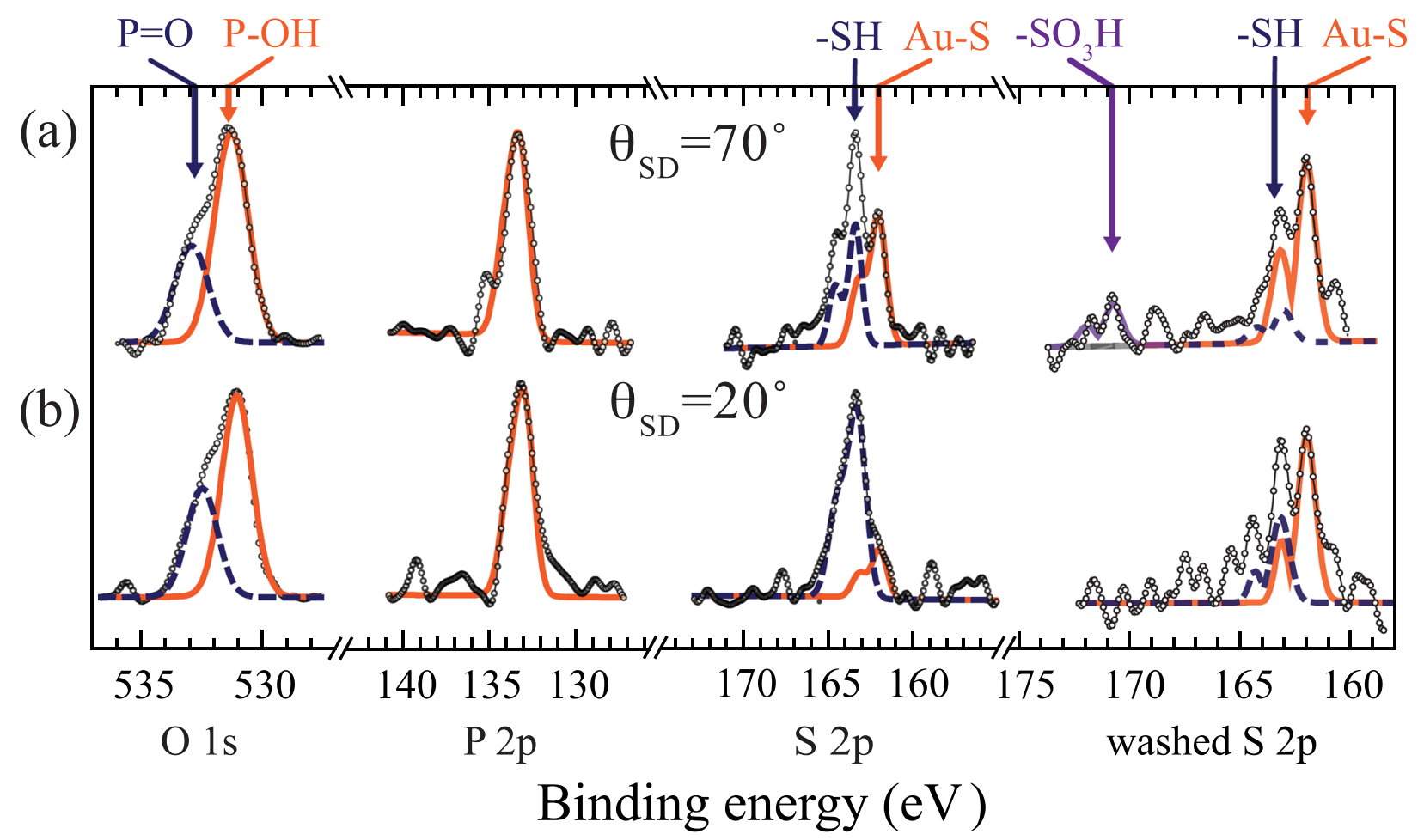

FIG. 3. Representative core-level O 1s, P 2p, and S 2p bands from MPPA-functionalized Au, and the S 2p bands obtained after a base-acid wash, obtained by XPS at photoelectron takeoff angles (a) $\theta_{\mathrm{SD}}=70^{\circ}$ and (b) $\theta_{\mathrm{SD}}=20^{\circ}$. 


\section{$\theta_{\mathrm{SD}}($ degree $)$}

(a)

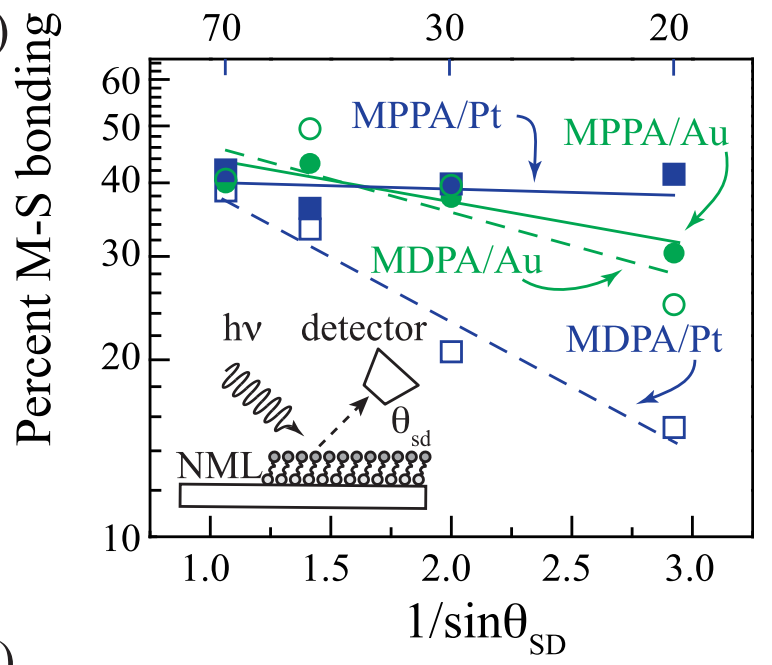

(b)

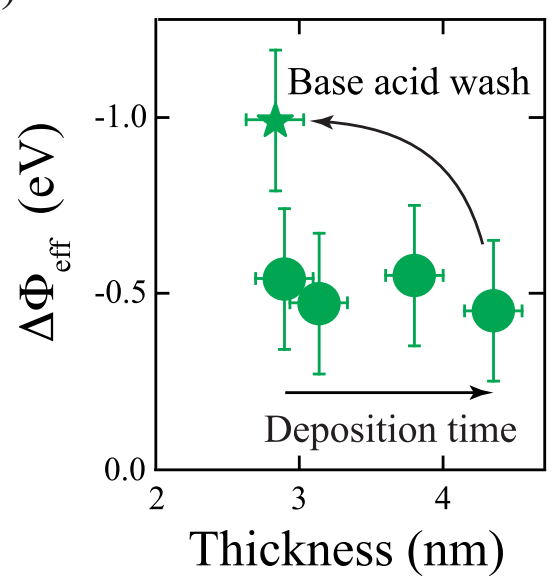

FIG. 4. (a) The $\mathrm{S} 2 \mathrm{p}$ sub-band fraction due to metal-sulfur bonding plotted as a function of $\theta_{\mathrm{SD}}$ for MPPA (filled symbols) and MDPA (unfilled symbols) on $\mathrm{Au}$ (circles) and Pt (squares). (b) Au work function shift plotted as a function of MPPA deposition. The nominal thickness is shown in the top abscissa. Also shown is the effect of a $\mathrm{NaOH}-\mathrm{HCl}$ wash (Star). Arrows indicate processing order.

However, this trend correlates with molecular length as well, ${ }^{41}$ which can influence the MNL morphology, as discussed here. Thus, further studies involving the direct measurement of MNL coverage are needed to isolate and understand the role of coverage on $\left|\Delta \Phi_{\text {eff }}\right|$.

Our experimental observations, as well as our theoretical calculations described earlier, confirm that mercaptanterminated organophosphonate MNLs consist of an intra-MNL bilayer. The physisorbed MPPA (MDPA) sublayer is weakly bound to the chemisorbed MPPA (MDPA) sub-layer via hydrogen bonding between phosphonic acid moieties that are proximally positioned, e.g., in a face-toface configuration, at the interface (Fig. 5(a)). Ordering of the sub-layers through van der Waals and hydrogen bonding between methylene and phosphonic acid, respectively, would increase with molecular length, which is verified in the Pt variable angle XPS (Fig. 4(a)). Such intra-MNL layering has been reported for carboxylic-acid based $\mathrm{MNLs}^{42}$ formed from high molecular concentrations. Our organophosphonate MNLs are formed from even higher concentrations than reported, thus favoring hydrogen bonding.

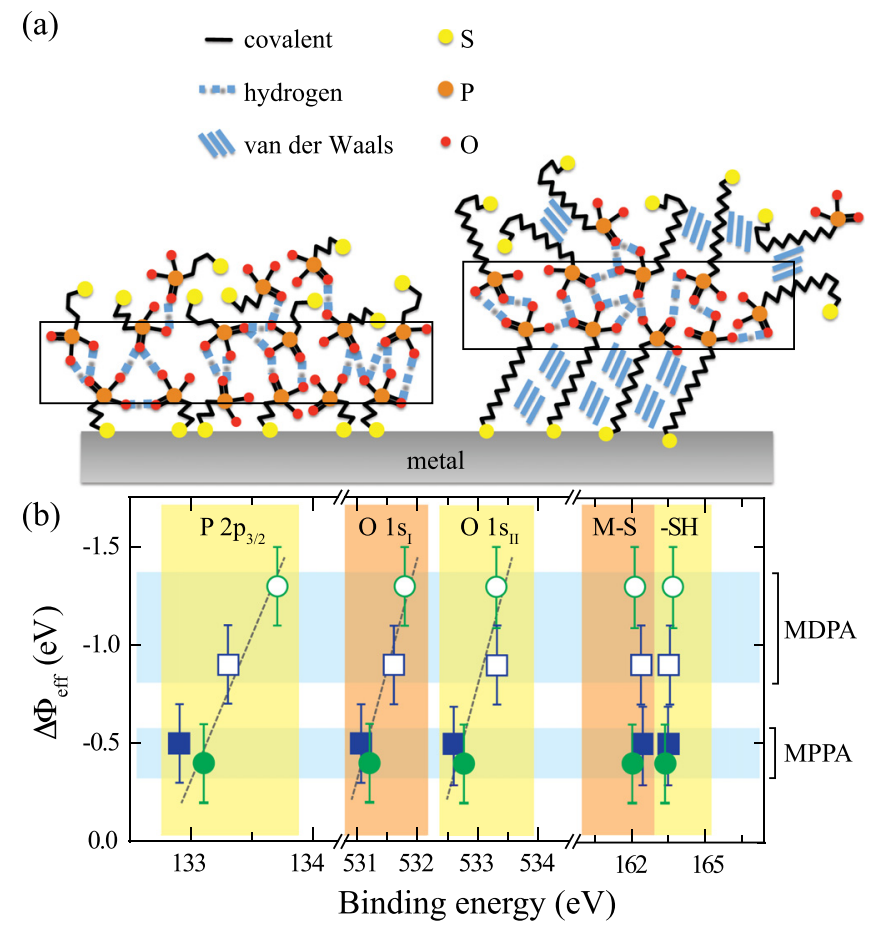

FIG. 5. (a) Schematic sketches depicting proposed interlayer bonding and morphology in the organophosphonate MNLs, due to hydrogen bonds (left) and a combination of hydrogen bonding with molecular orientation influenced by van der Waals forces (right). Boxed region indicates intra-MNL bonding contribution to $\Delta \Phi_{\text {eff }}$ (b) Metal work function shift $\Delta \Phi_{\text {eff }}$ plotted as a function of the core-level binding energies of $\mathrm{P} 2 \mathrm{p}_{3 / 2}, \mathrm{O} 1 \mathrm{~s}_{\mathrm{I}}$ (P-OH bonds), $\mathrm{O} 1 \mathrm{~s}_{\mathrm{II}}\left(\mathrm{P}=\mathrm{O}\right.$ bonds), and $\mathrm{S} 2 \mathrm{p}_{3 / 2}$ metal-sulfur bonds and free thiols for $\mathrm{Au}$ (circles) and Pt (squares) modified with MPPA (filled symbols) and MDPA (unfilled symbols). Lowering the binding energy of these signatures corresponds to increasing electron density around the non-metal atoms.

The pivotal role of intra-MNL interactions between phosphonic acid moieties is seen from the strong correlation between $\left|\Delta \Phi_{\text {eff }}\right|$ and the $\mathrm{P}$ and $\mathrm{O}$ core-level band positions for both MPPA and MDPA on both $\mathrm{Au}$ and Pt (see Fig. 5(b)). The Au-S and unbound mercaptan band positions remain unchanged, indicating that the effect of sulfur-based moieties in the physisorbed overlayer is negligible. Thus, our experimentally determined $\Delta \Phi_{\text {eff }}$ is attributable to intraMNL hydrogen bonding between the phosphonic acid moieties. The increase in $\mathrm{P} 2 \mathrm{p}_{3 / 2}$ and $\mathrm{O} 1 \mathrm{~s}$ binding energies with increasing molecular length suggests a greater electron deficiency in the phosphonic acid moieties of the longer molecule. Whereas randomly oriented physisorbed molecules have a net null effect, ${ }^{13,34}$ preferred molecular orientations would promote significantly higher $\Delta \Phi_{\text {eff. Both hydrogen }}$ bonding between phosphonic acid groups and van der Waals interactions between the methylene groups promote such ordering (see Fig. 5(a)). MNLs with longer organophosphonates allow for more van der Waals interactions and hence yield higher $\left|\Delta \Phi_{\text {eff }}\right|$.

In summary, weak intra-MNL chemical interactions can alter $\Phi_{\text {eff }}$ to an extent comparable to that obtained by covalent bonds at the MNL-metal interface. In particular, work function changes, induced by capping $\mathrm{Au}$ and Pt with MNLs of mercaptan-anchored organophosphonate molecules, cannot be explained solely in terms of a single layer of molecular dipoles at the interface. In fact, the electronic states of the moieties not bound to the metal bear a strong correlation 
with the observed shift, which can be amplified by using longer molecules. These findings suggest that unwanted multilayering could be a major source of lack of reproducibility of interfacial properties. However, controlling multilayering can also serve as an additional tool to tune the effective metal work function, and could alleviate the stringent requirements of forming an ordered monolayer for altering the metal work function.

We gratefully acknowledge support from the National Science Foundation ECCS Grant No. 1002282/301. University of Connecticut acknowledges support from National Science Foundation (NSF) and computational support through a NSF Teragrid Resource Allocation.

${ }^{1}$ G. D. Wilk, R. M. Wallace, and J. M. Anthony, J. Appl. Phys. 89, 5243 (2001).

${ }^{2}$ J. Robertson, Rep. Prog. Phys. 69, 327 (2006).

${ }^{3}$ S. A. DiBenedetto, A. Facchetti, M. A. Ratner, and T. J. Marks, J. Am. Chem. Soc. 131, 7158 (2009).

${ }^{4}$ M. Halik, H. Klauk, U. Zschieschang, G. Schmid, C. Dehm, M. Schütz, S. Maisch, F. Effenberger, M. Brunnbauer, and F. Stellacci, Nature 431, 963 (2004).

${ }^{5}$ I. De, D. Johri, A. Srivastava, and C. M. Osburn, Solid-State Electron. 44, 1077 (2000).

${ }^{6}$ A.-S. Duwez, J. Ghijsen, J. Riga, M. Deleuze, and J. Delhalle, J. Phys. Chem. B 101, 884 (1997).

${ }^{7}$ D. M. Alloway, A. L. Graham, X. Yang, A. Mudalige, R. Colorado, V. H. Wysocki, J. E. Pemberton, T. Randall Lee, R. J. Wysocki, and N. R. Armstrong, J. Phys. Chem. C 113, 20328 (2009).

${ }^{8}$ C. D. Zangmeister, L. B. Picraux, R. D. van Zee, Y. Yao, and J. M. Tour, Chem. Phys. Lett. 442, 390 (2007).

${ }^{9}$ C. Schmidt, A. Witt, and G. Witte, J. Phys. Chem. A 115, 7234 (2011).

${ }^{10}$ Y. C. Quintero, H. Zhu, and R. Ramprasad, J. Mater. Sci. 48, 2277 (2013).

${ }^{11}$ P. J. Hotchkiss, H. Li, P. B. Paramonov, S. A. Paniagua, S. C. Jones, N. R. Armstrong, J. Brédas, and S. R. Marder, Adv. Mater. 21, 4496 (2009).

${ }^{12}$ T. Schulmeyer, S. A. Paniagua, P. A. Veneman, S. C. Jones, P. J. Hotchkiss, A. Mudalige, J. E. Pemberton, S. R. Marder, and N. R. Armstrong, J. Mater. Chem. 17, 4563 (2007).

${ }^{13}$ X. Crispin, V. Geskin, A. Crispin, J. Cornil, R. Lazzaroni, W. R. Salaneck, and J.-L. Brédas, J. Am. Chem. Soc. 124, 8131 (2002).

${ }^{14}$ V. De Renzi, R. Rousseau, D. Marchetto, R. Biagi, S. Scandolo, and U. del Pennino, Phys. Rev. Lett. 95, 046804 (2005).

${ }^{15}$ L. Kornblum, Y. Paska, J. A. Rothschild, H. Haick, and M. Eizenberg, Appl. Phys. Lett. 99, 233508 (2011).
${ }^{16}$ L. Kornblum, Y. Paska, H. Haick, and M. Eizenberg, J. Phys. Chem. C 117, 233 (2013).

${ }^{17}$ D. D. Gandhi, A. P. Singh, M. Lane, M. Eizenberg, and G. Ramanath, J. Appl. Phys. 101, 084505 (2007).

${ }^{18}$ G. Ramanath, G. Cui, P. G. Ganesan, X. Guo, A. V. Ellis, M. Stukowski, K. Vijayamohanan, P. Doppelt, and M. Lane, Appl. Phys. Lett. 83, 383 (2003).

${ }^{19}$ G. Guerrero, J. G. Alauzun, M. Granier, D. Laurencin, and P. H. Mutin, Dalton Trans. 42, 12569 (2013).

${ }^{20}$ D. D. Gandhi, M. Lane, Y. Zhou, A. P. Singh, S. Nayak, U. Tisch, M. Eizenberg, and G. Ramanath, Nature 447, 299 (2007).

${ }^{21}$ P. J. O'Brien, S. Shenogin, J. Liu, P. K. Chow, D. Laurencin, P. H. Mutin, M. Yamaguchi, P. Keblinski, and G. Ramanath, Nat. Mater. 12, 118 (2012).

${ }^{22}$ P. K. Chow, Y. Cardona Quintero, P. O'Brien, P. H. Mutin, M. Lane, R. Ramprasad, and G. Ramanath, Appl. Phys. Lett. 102, 201605 (2013).

${ }^{23}$ S. Garg, A. Jain, C. Karthik, B. Singh, R. Teki, V. S. Smentkowski, M. W. Lane, and G. Ramanath, J. Appl. Phys. 108, 034317 (2010).

${ }^{24}$ O. Acton, G. Ting, H. Ma, J. W. Ka, H. Yip, N. M. Tucker, and A. K. Y. Jen, Adv. Mater. 20, 3697 (2008).

${ }^{25}$ O. Acton, M. Dubey, T. Weidner, K. M. O'Malley, T.-W. Kim, G. G. Ting, D. Hutchins, J. E. Baio, T. C. Lovejoy, A. H. Gage, D. G. Castner, H. Ma, and A. K.-Y. Jen, Adv. Funct. Mater. 21, 1476 (2011).

${ }^{26}$ D. Cahen and A. Kahn, Adv. Mater. 15, 271 (2003).

${ }^{27}$ D. A. Shirley, Phys. Rev. B 5, 4709 (1972).

${ }^{28}$ C. Bain and G. Whitesides, J. Phys. Chem. 93, 1670 (1989).

${ }^{29}$ G. Kresse and J. Furthmuller, Phys. Rev. B 54, 11169 (1996).

${ }^{30}$ P. C. Rusu and G. Brooks, J. Phys. Chem. B 110, 22628 (2006).

${ }^{31}$ J. P. Perdew, J. A. Chevary, S. H. Vosko, K. A. Jackson, M. R. Pederson, D. J. Singh, and C. Fiolhais, Phys. Rev. B 46, 6671 (1992).

${ }^{32}$ P. E. Blochl, Phys. Rev. B 50, 17953 (1994); G. Kresse and D. Joubert, ibid. 59, 1758 (1999).

${ }^{33}$ N. E. Singh-Miller and N. Marzari, Phys. Rev. B 80, 235407 (2009).

${ }^{34}$ M. Salmerón, S. Ferrer, M. Jazzar, and G. A. Somorjai, Phys. Rev. B 28, 6758 (1983).

${ }^{35}$ K. Wandelt, Appl. Surf. Sci. 111, 1 (1997).

${ }^{36}$ J. Amalric, P. H. Mutin, G. Guerrero, A. Ponche, A. Sotto, and J. Lavigne, J. Mater. Chem. 19, 141 (2009).

${ }^{37}$ Y. Chen, X.-J. Yang, B. Jin, L.-R. Guo, L.-M. Zheng, and X.-H. Xia, J. Phys. Chem. C 113, 4515 (2009).

${ }^{38}$ N. Gozlan, U. Tisch, and H. Haick, J. Phys. Chem. C 112, 12988 (2008).

${ }^{39}$ S. R. Puniredd, I. Platzman, R. T. Tung, and H. Haick, J. Phys. Chem. C 114, 18674 (2010).

${ }^{40}$ N. Gozlan and H. Haick, J. Phys. Chem. C 112, 12599 (2008).

${ }^{41}$ M. D. Porter, T. B. Bright, D. L. Allara, and C. E. D. Chidsey, J. Am. Chem. Soc. 109, 3559 (1987).

${ }^{42}$ R. Arnold, W. Azzam, A. Terfort, and C. Wöll, Langmuir 18, 3980 (2002). 